\title{
Analysis of the enhancer-blocking function of the TBS element from Petunia hybrida in transgenic Arabidopsis thaliana and Nicotiana tabacum
}

\author{
Stacy D. Singer $\cdot$ Jean-Michel Hily $\cdot$ \\ Kerik D. Cox
}

Received: 20 April 2011/Revised: 1 June 2011/Accepted: 13 June 2011/Published online: 26 June 2011

(C) Springer-Verlag 2011

\begin{abstract}
Transcriptional enhancers possess the ability to override the tissue-specificity and efficiency of nearby promoters, which is of concern when generating transgenic constructs bearing multiple cassettes. One means of preventing these inappropriate interactions is through the use of enhancer-blocking insulators. The 2-kb transformation booster sequence (TBS) from Petunia hybrida has been shown previously to exhibit this function when inserted between an enhancer and promoter in transgenic Arabidopsis thaliana. In this study, we attempted to further characterize the ability of this fragment to impede enhancer-promoter interference through an analysis of transgenic Arabidopsis and Nicotiana tabacum lines bearing various permutations of the $T B S$ element between the cauliflower mosaic virus (CaMV) $35 \mathrm{~S}$ enhancer and an assortment of tissue-specific promoters fused to the $\beta$-glucuronidase (GUS) reporter gene. The full-length TBS fragment was found to function in both orientations, although to a significantly lesser degree in the reverse orientation, and was operational in both plant species tested. While multiple deletion fragments were found to exhibit activity, it appeared that several regions of the TBS were required for maximal enhancer-blocking function. Furthermore, we found that this element exhibited promoter-like activity, which has implications in terms of possible mechanisms behind its ability to impede enhancer-promoter communication in plants.
\end{abstract}

Communicated by R. Schmidt.

S. D. Singer · J.-M. Hily · K. D. Cox $(\varangle)$

Department of Plant Pathology and Plant-Microbe Biology,

New York State Agricultural Experiment Station,

Cornell University, Geneva, NY 14456, USA

e-mail: kdc33@cornell.edu
Keywords TBS element - Enhancer-blocking insulator · CaMV $35 S$ promoter/enhancer $\cdot$ Arabidopsis thaliana . Nicotiana tabacum - Enhancer-promoter interference

\section{Introduction}

The use of plant biotechnology is a necessity for both basic research and the enhancement of agronomic traits in crop species. While the majority of transgenic research is concerned with the improvement of a single trait, it is often the case that crops in field conditions must cope with a relatively large array of challenges that could be dealt with using genetic manipulation. Therefore, the implementation of broader approaches to improve the performance of several traits simultaneously using transformation vectors that harbor multiple transcriptional units is becoming more commonplace. This is often accomplished using a strong, constitutive promoter/enhancer to drive the expression of the selectable marker combined with tissue-, organ- or developmental stage-specific promoters to initiate the expression of transgenes solely in targeted tissues. However, this strategy is often problematic due to the positionand orientation-independent ability of enhancers to trigger enhancer-promoter interference and disturb the expression of the transgene of interest. This phenomenon is particularly prevalent in transgenic plants harboring constructs in which the enhancer from the CaMV $35 \mathrm{~S}$ promoter (Odell et al. 1988) is in relatively close proximity to the promoter of another transgene, and results in both a loss of specificity and an increase in the level of expression induced by the transgenic promoter (Hily et al. 2009; Zheng et al. 2007). Interestingly, this enhancer-mediated activation of proximal promoters appears to be a fairly common property of a wide range of enhancers, and as a result, has incited interest 
in the development of strategies with which to prevent such interactions (Gudynaite-Savitch et al. 2009; Hily et al. 2009; Singer et al. 2010b).

Although mechanisms exist in eukaryotic genomes to prevent inappropriate enhancer-promoter interactions (reviewed by Kadauke and Blobel 2009), transgenic constructs lack this ability and thus require additional means with which to block enhancer-mediated activation of proximal promoters. Since not all enhancers possess the same activation potential (Gudynaite-Savitch et al. 2009), a possible solution to this problem is to generate transgenic constructs utilizing enhancers that do not have an effect on the tissue-specificity or strength of nearby promoters. However, promoters vary in their responsiveness to the activation potential of each enhancer, suggesting that one single enhancer would not necessarily solve the problem. Another possible approach with which to prevent enhancer-promoter crosstalk is to physically separate enhancers and promoters within the transgenic construct (Jagannath et al. 2001). Unfortunately, since both the strength of the enhancer and the sensitivity of the particular target promoter have an effect on the exact length of separation required to inhibit enhancer-mediated mis-expression, the precise length of spacer sequence required will differ from construct to construct, which further complicates this strategy. Enhancer-promoter interference can also be obstructed through the use of enhancer-blocking insulators which inhibit enhancer-promoter communication when situated between the two. A number of these elements have been characterized in metazoan systems and include the gypsy retrotransposon element (Geyer et al. 1986), the cHS4 insulator from the chicken $\beta$-globin locus (Chung et al. 1993) and the Drosophila scs/scs' paired elements (Kellum and Schedl 1992). While such extensive research with respect to enhancer-blocking insulators has not been carried out in plant species as of yet, there has recently been a surge of interest in this topic as it may provide a means for minimizing enhancer-promoter crosstalk during plant transformation with composite vectors (reviewed by Singer et al. 2011). For example, the 2-kb transformation booster sequence $(T B S)$ from Petunia hybrida was previously found to impede activation of the flower-specific AGAMOUS second intron-derived promoter $(A G I P)$ in vegetative tissues by the partially duplicated $35 S$ enhancer when situated between the two in transgenic Arabidopsis (Hily et al. 2009). However, the mean values by which the $T B S$ carries out its function remain unclear as of yet.

To date, several models explaining the mechanism behind enhancer-blocking insulator function in animal systems have been proposed. One such model suggests that they provide a steric effect by separating chromatin into topologically distinct domains through the binding of proteins which form clusters localized at the nuclear periphery, resulting in loops of DNA across which enhancer-promoter interactions cannot take place (Gaszner and Felsenfeld 2006). Alternatively, the binding of proteins to the insulator sequence could result in a physical blockage of an activating signal, such as histone modification or intergenic transcription initiated at the enhancer and progressing toward the target promoter (reviewed by Wallace and Felsenfeld 2007). It has also been suggested that enhancer-blocking insulators may function as decoy promoters by directly interacting with the enhancer or through the attenuation of a putative signal that progresses from enhancer to promoter, thus inhibiting communication between the enhancer and target promoter (reviewed by Raab and Kamakaka 2010). Since evidence exists to support each of these models, it is probable that one single model is not applicable to all enhancer-blocking activity.

In this study, we endeavored to further characterize the mechanism through which the TBS fragment acts as an enhancer-blocking insulator in plants. First, the full-length $T B S$ was tested in both orientations for its ability to block activation of the petal- and stamen-specific PISTILLATA promoter $(P I p)$ by the partially duplicated CaMV $35 S$ enhancer in the leaves of transgenic Arabidopsis. Similarly, a series of $5^{\prime}$ and $3^{\prime}$ deletions of the $T B S$ were inserted between the enhancer and target promoter in an attempt to narrow down the precise region required for enhancerblocking activity. Furthermore, the activity of the TBS fragment was assayed in transgenic Nicotiana tabacum to ascertain its functionality in multiple plant species. To gain insight into the mechanism behind its function, we tested its ability to act as a promoter by fusing the full-length $T B S$ directly to the GUS reporter gene and assaying the resulting transgenic Arabidopsis plants for GUS expression.

\section{Materials and methods}

\section{Plasmid constructs}

Schematic representations of the transforming constructs utilized in this study are shown in Fig. 1. All vectors were produced using standard protocols and include pBINplus (van Engelen et al. 1995) with an inserted PZP-RSC1 multiple cloning site (Hajdukiewicz et al. 1994) as a background and verified by sequencing prior to transformation. A positive control vector for constitutive GUS expression was generated that contained the petal- and stamen-specific PI promoter fragment (PIp) upstream of the GUSAint sequence (Ohta et al. 1990) fused to the nopaline synthase transcriptional terminator (nos- $t$ ) in a head-to-head orientation with a cassette comprising the partially duplicated $35 \mathrm{~S}$ promoter (referred to as $35 \mathrm{~S}$ throughout the text; Kay et al. 1987), enhanced green 
fluorescent protein (eGFP) coding sequence and nos- $t$ (p1). The $35 S$ and PIp sequences were separated by $138 \mathrm{bp}$ of intervening vector sequence. This was executed by inserting the GUSAint::nos- $t$ cassette into the BamHI/EcoRI site of the background vector and the 35S::eGFP::nos- $t$ cassette into the PI-PspI site of the PZP-RSC1 multiple cloning site. A fully functional $3^{\prime}$ 378-bp fragment of the PIp, which terminates immediately upstream of the translational start codon and drives expression specifically in petals and stamens, was cloned from Arabidopsis ecotype col-0. Primers PIfwdXbaI (TCT AGA CAC ATG CAA AGA GTG TCA TTA AGC A) and PIrevBamHI (GGA TCC CTT TCT CTC TCT ATC TCT CTT TCT CAA TTT T) were utilized for amplification using Platinum PCR SuperMix High Fidelity according to the manufacturer's instructions (Invitrogen, San Diego, CA, USA). The primers were based on those designed by Honma and Goto (2000), but included restriction sites at their $5^{\prime}$ ends to enable cloning into the XbaI/BamHI site immediately upstream of the GUSAint::nos- $t$ cassette. A positive control vector for PIp-conferred petal- and stamen-specific expression was generated by digesting $\mathrm{p} 1$ with $P I-P s p I$ and re-ligating to remove the 35S::eGFP::nos- $t$ cassette (PIp::GUS). A positive control vector for $35 S$-specific eGFP expression was produced by digesting PIp::GUS with $X b a \mathrm{I}$ and EcoRI, end-filling, and re-ligating to remove the PIp::GUS::nos- $t$ cassette. The resulting vector was subsequently digested with PI-PspI and the $35 S:: e G F P::$ $n o s-t$ cassette from $\mathrm{p} 1$ was inserted (35S::GFP).

The 2,018-bp TBS fragment from $P$. hybrida cultivar V26 (Hily et al. 2009; GenBank accession EU864306), which we will subsequently refer to as the full-length TBS sequence, was inserted into the EcoRI site of the pAUX3132 vector (Goderis et al. 2002). This element has been found to function previously in one orientation (reverse polarity relative to GenBank accession EU864306) to block enhancer-promoter interactions between the $35 \mathrm{~S}$ enhancer and AGIP in Arabidopsis (Hily et al. 2009). To test whether the sequence was functional in both orientations and with a novel promoter, the full-length $T B S$ fragment was subsequently introduced into the $I-C e u I$ site of p1 between the 35S::eGFP::nos-t and PIp::GUSAint:: $n o s-t$ cassettes in forward (p1-TBSF) and reverse orientations (p1-TBSR). A control vector for insert sequence length was also generated, which included a putatively 'inert' sequence of similar size to the full-length $T B S$ between the GFP and GUS cassettes in $\mathrm{p} 1$. This was accomplished by first cloning a 2,158-bp region of the Atcopia28-like fragment from genomic Arabidopsis DNA with primers AtcopiaF2HindIII (AAG CTT GTA GTG AGT TGA TGT TAT GAA TGA) and AtcopiaR2HindIII (AAG CTT AAC ATG TTT CTT GCT CCA TAT TAC A) and inserting the sequence into the HindIII site of
pAUX3132. The resulting vector was digested with I-CeuI and the Atcopia fragment was introduced into the same site of p1 (p1-Spacer1). A similar length-control vector was generated by inserting a 3,967-bp NcoI fragment of bacteriophage $\lambda$ (nucleotides 23,901-27,868) between the $35 S$ enhancer and PIp of $\mathrm{p} 1$, which had been shown in previous studies to not impede promoter/enhancer interactions (Hily et al. 2009; Singer et al. 2010b), into pAUX3132, and subsequently introducing the fragment into the $I-C e u I$ site of p1 (p1-Spacer2).

To assay the functionality of various portions of the TBS fragment in the forward orientation, a series of $5^{\prime}$ and $3^{\prime}$ deletions were devised. In each case, a vector containing the full-length TBS in a pAUX3132 background was digested with a specific set of restriction enzymes, endfilled, and re-ligated to generate the deletion. The resulting vectors were then digested with $I-C e u I$ and the fragments were inserted into p1 between the $35 S:: e G F P:: n o s-t$ and PIp::GUSAint::nos- $t$ cassettes. The enzymes StuI and SalI were utilized to produce a $5^{\prime} 827-b p$ fragment of the TBS (p1-TBSdel1). BlpI and SalI, along with AgeI and StuI, were used to generate a 465-bp fragment comprising the middle of the TBS (p1-TBSdel2), AgeI and BlpI were used to create a $3^{\prime} 726-\mathrm{bp}$ fragment of the TBS (p1-TBSdel3), BlpI and SalI were used to produce a $5^{\prime}$ 1,292-bp fragment of the TBS (p1-TBSdel4), and AgeI and StuI were used to produce a $3^{\prime} 1,191-\mathrm{bp}$ fragment of the TBS (p1-TBSdel5).

To test the effectiveness of the full-length forward-oriented TBS fragment as an enhancer-blocking insulator within a different species, as well as with another promoter, we generated further constructs in which the PIp was replaced by the phloem-specific SUS1 promoter from Arabidopsis (AtSUS1p; Sadeghi et al. 2007). To generate a positive control vector for phloem-specific expression, the PIp::GUS vector was digested with $\mathrm{XbaI}$ and $\mathrm{BamHI}$ and a 1,515-bp fragment of the AtSUS1p terminating directly upstream of the translational start codon [amplified using primers AtSUS1F1XbaI (TCT AGA GAT ATC ATT TCA TAT CAT CA) and AtSUS1R1BamHI (GGA TCC AAA AGA GAC GCA GAA AAC AG)] was inserted in its place (AtSUS1p::GUS). A positive control vector for constitutive GUS expression (akin to p1) was generated by introducing the $35 S:: e G F P:: n o s-t$ cassette into the PI-PspI site of the AtSUS1p::GUS vector (p2). The function of the TBS element was assayed by inserting the TBS fragment in the forward orientation into the I-CeuI site of p2 (p2-TBSF). A vector to control for the length of the insert sequence was generated by inserting the 3,967-bp NcoI fragment from bacteriophage $\lambda$ into the $I-C e u I$ site of p2 (p2-Spacer2).

To test the full-length TBS element for promoter activity, the 2,018-bp fragment was cloned into pAUX3166 (Goderis et al. 2002) and subsequently inserted into the Asc I site just upstream of a promoterless GUSAint:: 
nos- $t$ cassette in both the forward (TBSF::GUS) and reverse (TBSR::GUS) orientations.

\section{Transformation of Arabidopsis and N. tabacum}

Constructs were introduced into Agrobacterium tumefaciens strain GV3101 via electroporation and the resulting bacteria were utilized to transform Arabidopsis thaliana ecotype col-0 and N. tabacum cultivar NC95 (a gift from Dr. Georg Jander, Boyce Thompson Institute for Plant Research, Cornell University). Transformation of Arabidopsis was conducted using the floral dip method (Clough and Bent 1998), which has been shown previously to result in the introduction of a single T-DNA insert in more than $50 \%$ of transgenic lines (Alonso et al. 2003; Rosso et al. 2003). Subsequently, surface-sterilized seeds were plated on standard Murashige and Skoog (MS) media (Murashige and Skoog 1962) containing $60 \mathrm{mg} / \mathrm{L}$ kanamycin to allow the selection of transformants. Transformation of tobacco leaves was carried out as described by Horsch et al. (1985) and transformed shoots were selected on standard MS medium containing $1 \mathrm{mg} / \mathrm{L}$ benzyl adenine (BA), $300 \mathrm{mg} / \mathrm{L}$ timentin and $100 \mathrm{mg} / \mathrm{L}$ kanamycin. Rooting was subsequently induced by plating on the same medium lacking BA. All transgenic plants utilized in this study were phenotypically normal primary transformants.

Histochemical staining and fluorometric assays of GUS activity

Histochemical assays for GUS staining were carried out as described by Jefferson et al. (1987). Leaf and floral tissue from a selection of transgenic Arabidopsis lines bearing each vector, respectively, as well as petiole tissue from transgenic tobacco, were incubated in $1 \mathrm{mM} 5$-bromo-4chloro-3-indolyl- $\beta$-D-glucuronide (X-gluc; in $100 \mathrm{mM}$ phosphate buffer, $\mathrm{pH} 7.0,10 \mathrm{mM}$ EDTA, $0.5 \mathrm{mM}$ potassium ferrocyanide and $0.1 \%$ Triton $\mathrm{X}-100$ ) at $37^{\circ} \mathrm{C}$ for $24 \mathrm{~h}$. Tissues were subsequently depigmented in a series of $70 \%$ ethanol washes and images were acquired using an Olympus BX50 light microscope (Olympus America Inc., Center Valley, PA, USA) outfitted with a SPOT Idea digital camera (Diagnostic Instruments Inc., Sterling Heights, MI, USA). The overall levels of GUS staining observed in the leaves of each transgenic line were compared with those of lines containing the "insertless" control vector, $\mathrm{p} 1$, and were scored as similar or reduced on this basis.

Fluorometric assays of GUS activity were carried out to quantify GUS protein levels as described by Hily et al. (2009). Briefly, three 4-week-old leaves were analyzed from a minimum of 12 independent Arabidopsis lines containing each vector tested, respectively, as well as an untransformed control. Concentrations of methylumbelliferone (MU)
Fig. 1 Schematic diagram of constructs utilized in this study. All vectors shown are present in a pBINPLUS background. Arrows indicate the orientation of genetic elements. PIp-based plasmids utilized in the characterization of the enhancer-blocking activity of the TBS element are shown in (a). Vector $35 \mathrm{~S}$ ::GFP is a negative control lacking the GUS reporter gene, while vector PIp::GUS is a control plasmid for PIp-driven petal- and stamen-specific GUS expression. Construct $\mathrm{p} 1$ is the base vector, which contains the $35 S:: e G F P$ and PIp::GUS cassettes in opposite directions, and is a positive control for $35 S$ enhancer-mediated constitutive GUS expression. Each remaining vector is a derivative of $\mathrm{p} 1$, bearing different DNA sequences between the $35 S$ promoter and PIp. AtSUS1p-based vectors utilized in the analysis of enhancer-blocking activity of the TBS element in tobacco are displayed in (b). Vector AtSUS1p::GUS is a control plasmid for AtSUS1p-driven phloem-specific GUS expression, while $\mathrm{p} 2$ is the base of the remaining vectors containing the 35S::eGFP and AtSUS1p::GUS cassettes in a head-to-head orientation and is a positive control for $35 S$ enhancer-mediated constitutive GUS expression. Constructs p2-TBSF and p2-Spacer2 are derivatives of $\mathrm{p} 2$ bearing the $T B S$ fragment in the forward orientation and a 3,967-bp control $\lambda$ spacer fragment, respectively, between the $35 S$ enhancer and AtSUS1p. Vectors designed to test the ability of the $T B S$ element to initiate transcription are shown in (c). Plasmids TBSF::GUS and TBSR::GUS contain the TBS element in forward and reverse orientations, respectively, fused directly to the GUSAint reporter gene. $35 S$, CaMV $35 S$ partially duplicated promoter; $e G F P$, enhanced green fluorescent protein; nt, nopaline synthase transcriptional terminator; PIp, PISTILLATA petal- and stamen-specific promoter; GUSAint, $\beta$-glucuronidase reporter gene containing an intron; AtSUS1p, Arabidopsis sucrose synthase 1 phloem-specific promoter

generated were established using the linear regression slopes of fluorescence emitted by a series of MU standards. Concentrations of total protein in each sample were determined using the Bio-Rad protein assay system (Bio-Rad Laboratories, Hercules, CA, USA) with bovine serum albumin as a standard. Each sample was assayed in duplicate and resulting GUS activities were expressed as the mean value of pmol MU produced minute ${ }^{-1} \mathrm{mg}$ total protein $^{-1}$ (pmol MU/ min $\mathrm{mg}$ ). Statistical analyses were conducted between insert-bearing lines and 'insertless' p1 lines using the MannWhitney test for non-parametric data and differences were considered significant at $P \leq 0.05$.

Semi-quantitative RT-PCR and quantitative real-time RT-PCR analysis of GUS expression

Total RNA was extracted from Arabidopsis tissue using the E.Z.N.A. Plant RNA Mini Kit according to the manufacturer's instructions (Omega Bio-Tek, Norcross, GA, USA). Contaminating DNA was subsequently removed with the TURBO DNA-free system (Ambion, Austin, TX, USA). In an attempt to determine whether the TBS fragment was capable of initiating transcription of a downstream reporter gene, semi-quantitative RT-PCR assays were conducted on $450 \mathrm{ng}$ total RNA from a mixture of leaf and floral tissues of two independent TBSF::GUS and TBSR::GUS lines (which contain the TBS element fused to GUS in the 
(a)

$\begin{array}{lll}\text { 35S::GFP } & \text { 2018-bp TBS forward orientation } \\ \text { p1-TBSF } & \text { 2018-bp TBS reverse orientation } \\ \text { p1-TBSR } & \text { 5' 827-bp TBS fragment } \\ \text { p1-TBSdel1 } & \text { 3id 465-bp TBS fragment } \\ \text { p1-TBSdel2 } & \text { 3' 726-bp TBS fragment } \\ \text { p1-TBSdel3 } & \text { 3' 1292-bp TBS fragment } \\ \text { p1-TBSdel4 } & \text { 2158-bp Atcopia28-like spacer DNA } \\ \text { p1-TBSdel5 } & \text { 3967-bp lambda spacer DNA } \\ \text { p1-Spacer1 } & \end{array}$

(b)

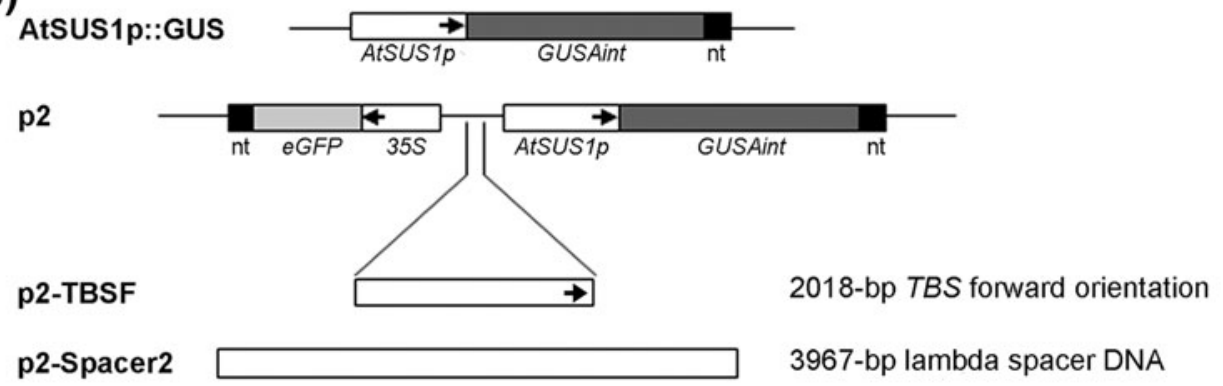

(c)

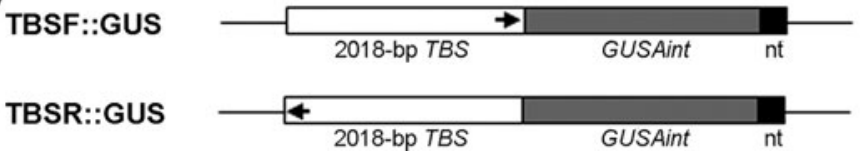

forward and reverse orientations), respectively, along with an untransformed control. First-strand cDNA synthesis was carried out using the Superscript III first-strand cDNA synthesis kit (Invitrogen) and an oligo-dT primer in a final volume of $10 \mu \mathrm{l}$. Subsequent PCR assays were executed using $1 \mu \mathrm{l}$ of the resulting cDNA as template with HotStart GoTaq polymerase (Promega, Madison, WI, USA) in a final volume of $25 \mu$. Primers GUSintF1 (CGT TGG TGG AAA GCG CGT TAC) and GUSintR3 (CTG CGA TGG ATT CCG GCA TAG) were used in an attempt to amplify a 614-bp fragment of GUS-specific cDNA. These primers anneal on either side of the intronic region of the GUSAint reporter gene and thus allowed for the differentiation of genuine products derived from cDNA and those resulting from any remaining genomic DNA contamination. An $E F 1 \alpha$-specific product of $630 \mathrm{bp}$ was used as an internal control and was amplified with primers EF $1 \alpha \mathrm{F}$ and $\mathrm{EF} 1 \alpha \mathrm{R}$ (Hily and Liu 2009). Thermal parameters for the amplification of $G U S$-specific product were $95^{\circ} \mathrm{C}$ for $2 \mathrm{~min}$, followed by 28 cycles of $95^{\circ} \mathrm{C}$ for $30 \mathrm{~s}, 62^{\circ} \mathrm{C}$ for $30 \mathrm{~s}$, and 
$72^{\circ} \mathrm{C}$ for $1 \mathrm{~min}$, with a final elongation step of $72^{\circ} \mathrm{C}$ for $5 \mathrm{~min}$. The same general conditions were used to amplify $E F 1 \alpha$-specific product with the exception of the annealing temperature, which was $58^{\circ} \mathrm{C}$, and the utilization of 22 cycles. PCR products were resolved on a $1.5 \%$ agarose gel and visualized using ethidium bromide.

Quantitative real-time RT-PCR analysis of GUS expression was carried out on total RNA from the leaves of three independent transgenic Arabidopsis lines bearing each of the constructs, respectively, along with an untransformed control. For each line, $20 \mathrm{ng}$ total RNA were utilized with the IScript One-Step qRT-PCR kit according to the manufacturer's recommendations (Bio-Rad Laboratories) in a final volume of $15 \mu$ l. Assays were performed in triplicate on an iCycler IQ5 detection system (Bio-Rad Laboratories) using primers GUSRTF2 and GUSRTR2 (Hily et al. 2009) to amplify a 183-bp fragment of the GUS transcript and actin-specific primers (Charrier et al. 2002) to amplify a 108-bp fragment of the internal standard transcript, Actin2. Reactions including no template and no reverse transcriptase were included in each trial. Thermal parameters for RT-PCR amplification were $50^{\circ} \mathrm{C}$ for $10 \mathrm{~min}, 95^{\circ} \mathrm{C}$ for $5 \mathrm{~min}$, and 40 cycles of $95^{\circ} \mathrm{C}$ for $10 \mathrm{~s}$ and $60^{\circ} \mathrm{C}$ for $30 \mathrm{~s}$. Dissociation curves were generated to ascertain that only a single product was produced in each case. Relative levels of gene expression were deduced from standard curves produced using a set of serial dilutions. All GUS expression data represent the mean values normalized to those of Actin2.

\section{Bioinformatic analyses}

To further elucidate the mechanism driving the enhancerblocking activity of the $T B S$, the fragment was scanned for possible promoter regions. In silico prediction of putative promoter regions was carried out using the Neuronal Network Promoter Prediction program (http://www.fruitfly. org/seq_tools/promoter.html; Reese 2001). A minimum cutoff score of 0.8 was utilized for the analysis.

\section{Results}

The TBS element inhibits $35 S$ enhancer-mediated activation of PIp-driven GUS expression in non-target tissues to a higher degree in the forward orientation

To determine whether the TBS element is capable of inhibiting activation of the PIp in an orientation-independent manner, vectors were generated in which the 35S::eGFP and PIp::GUS cassettes were present in a headto-head orientation with the TBS inserted between the two in both orientations, respectively (Fig. 1a). Leaves and flowers from independent lines containing each of the vectors, respectively, were analyzed histochemically for GUS staining (Fig. 2a). As expected, negative control lines containing the $35 S:: e G F P$ cassette, but no PIp::GUS cassette (35S::GFP), exhibited no GUS staining in any tissue type ( $n=6$, where $n$ is the number of independent transgenic lines analyzed). Also as anticipated, control lines for $P I p$-specific petal- and stamen-specific expression containing the PIp::GUS cassette, but no 35 S::eGFP cassette (PIp::GUS), displayed no GUS staining in leaf tissues but were found to express GUS in the petals and stamens of flowers $(n=16)$. All positive control lines for the $35 S$ enhancer-mediated activation of PIp::GUS in non-target tissues in which the $35 S:: e G F P$ and PIp::GUS cassettes were fused in a head-to-head orientation (p1) exhibited relatively high levels of GUS staining in a constitutive manner in all tissue types analyzed $(n=35)$. Similarly, insertion of an 'inert' $2-\mathrm{kb}$ spacer sequence derived from the Atcopia28-like sequence from Arabidopsis between the $35 S$ enhancer and PIp (p1-Spacer1) did not impede enhancer-promoter interference and constitutive GUS staining was observed in both the leaves and flowers of $95 \%$ of lines $(n=37)$. Conversely, $70 \%$ of lines-bearing vectors in which the TBS element was introduced in the forward orientation (p1-TBSF) exhibited blocking of $35 \mathrm{~S}$ enhancer-mediated activation of the PIp in non-target tissues, and GUS staining was only observed in petal and stamen tissues $(n=33)$. Inclusion of the TBS in the reverse orientation at the same site (p1-TBSR) resulted in $74 \%$ of lines exhibiting a similar inhibition of enhancer-promoter interactions, but possibly to a lesser degree $(n=35$; Fig. 2a).

Both $5^{\prime}$ and $3^{\prime}$ fragments of the $T B S$ element confer enhancer-blocking activity in transgenic Arabidopsis

To gain insight into which region of the $T B S$ is required for its insulator-blocking activity, we generated a series of vectors-bearing insertions of various $5^{\prime}$ and $3^{\prime}$ deletions of this element in the forward orientation between the 35S::eGFP and PIp::GUS cassettes (Fig. 1a). Histochemical analyses of leaf tissues indicated that the majority of lines bearing the $5^{\prime} 827$ bp of the TBS (p1-TBSdel1; 64\%; $n=25$ ), the $5^{\prime} 1,292$ bp of the TBS (p1-TBSdel4; 61\%; $n=18)$ and the $3^{\prime} 1,191 \mathrm{bp}$ of the TBS (p1-TBSdel5; 55\%; $n=38$ ) exhibited lower levels of GUS staining in leaf tissues compared with the positive control p1 lines. However, the level of staining in p1-TBSdel1 leaves appeared to be higher than the p1-TBSdel4 and p1-TBSdel5 lines. Conversely, only $24 \%$ of transgenic lines bearing the mid 465 bp of the TBS (p1-TBSdel2; $n=50$ ) and $35 \%$ of lines containing the $3^{\prime} 726 \mathrm{bp}$ of the TBS (p1-TBSdel3; $n=20$ ) exhibited reduced levels of GUS staining in leaf tissues 
Fig. 2 Histochemical GUS staining in the leaves of transgenic Arabidopsis lines. a Images display leaves and flowers of representative Arabidopsis lines transformed with various constructs utilized to test the ability of the TBS element in forward and reverse orientations to block constitutive activation of the petal- and stamen-specific PIp in non-target tissues by the $35 \mathrm{~S}$ enhancer. b Representative leaves from transgenic Arabidopsis lines-bearing constructs testing the enhancerblocking ability of various deletions of the TBS element (a)

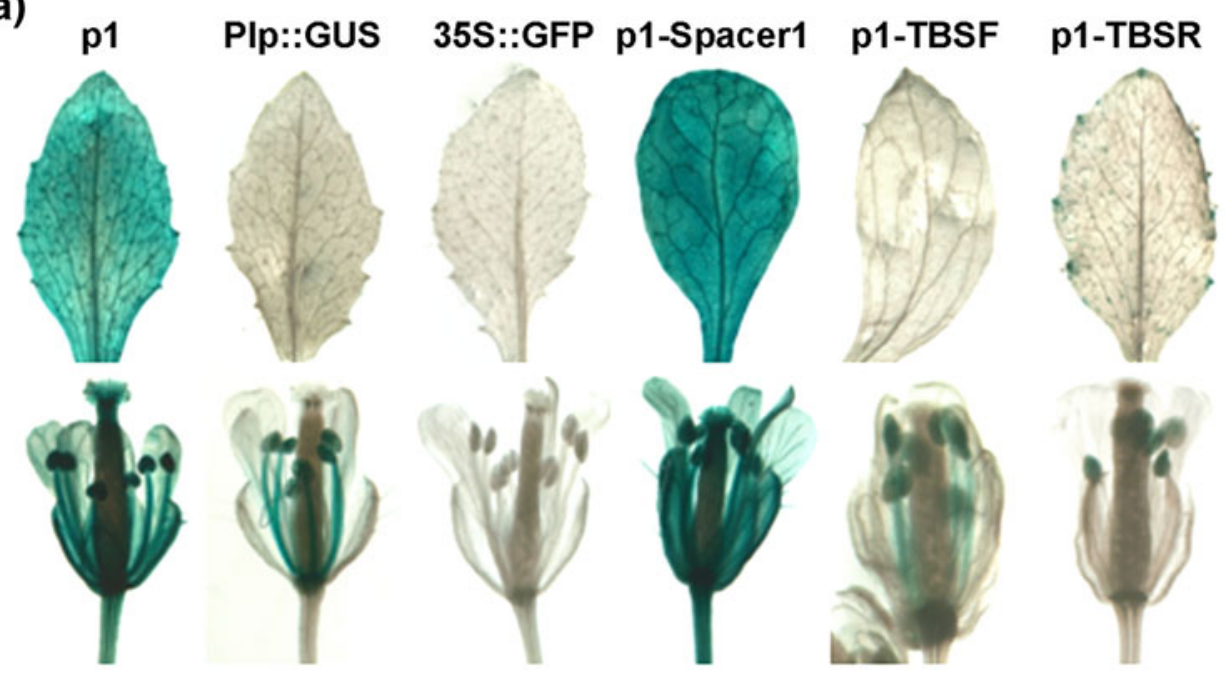

(b)

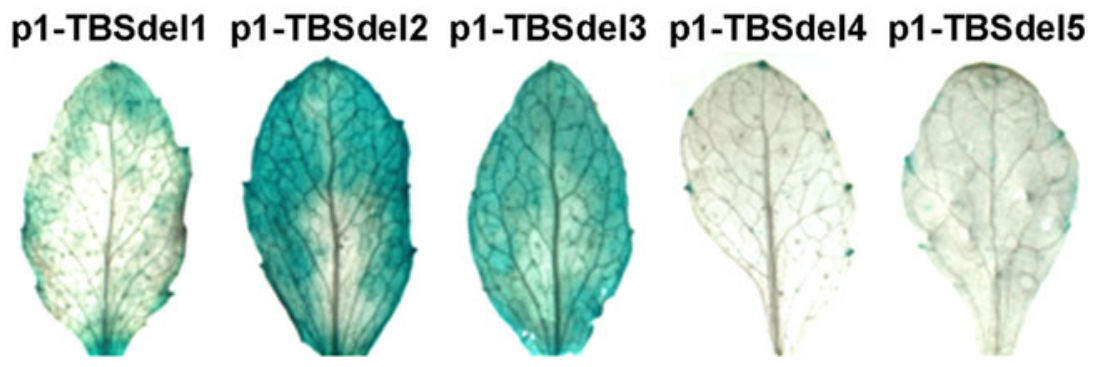

compared with the positive controls. Furthermore, the levels of GUS staining in the leaves of p1-TBSdel2 and p1TBSdel3 lines exhibiting reductions in GUS activity appeared to be higher than in p1-TBSdel1, p1-TBSdel4 and p1-TBSdel5 lines (Fig. 2b).

Quantification of enhancer-blocking activity in various transgenic Arabidopsis lines

Fluorometric assays for GUS activity were carried out on a selection of independent transgenic lines to quantify the levels of GUS protein in the leaves (Fig. 3a). As expected, only minimal levels of background GUS activity were detected in PIp::GUS lines, which lack the 35S::eGFP cassette $(35.1 \pm 6.8 \mathrm{pmol} \mathrm{MU} / \mathrm{min} \mathrm{mg}$ protein), resembling the basal activity displayed by untransformed lines (35.2 $\pm 5.3 \mathrm{pmol} \mathrm{MU} / \mathrm{min} \mathrm{mg}$ protein). Conversely, both p1 lines, which lack any insert sequence between the $35 S:: e G F P$ and PIp::GUS cassettes $(4,679.2 \pm$ 2,308.3 pmol MU/min mg protein), and p1-Spacer1 lines, which possess a control 'inert' 2-kb spacer sequence between the two cassettes $(3,337.0 \pm 1,101.2 \mathrm{pmol} \mathrm{MU} / \mathrm{min} \mathrm{mg}$ protein), displayed relatively high levels of GUS protein in leaf tissues. These values were not significantly different from one another $(P>0.05)$, indicating that the $35 S$ enhancer is able to effectively activate the petal- and stamenspecific PIp in non-target tissue even in the presence of an intervening $2-\mathrm{kb}$ fragment. Lines possessing either the fulllength $T B S$ in the forward (p1-TBSF) or reverse (p1-TBSR) orientations generated significantly lower $(P \leq 0.001)$ levels of GUS protein in their leaves $(136.9 \pm 57.9$ and $1,666.6 \pm 590.4 \mathrm{pmol} \mathrm{MU} / \mathrm{min} \mathrm{mg}$ protein, respectively) compared with the background $\mathrm{p} 1$ vector. While this suggests that the TBS is functional in both orientations, these values were significantly different from one another $(P=0.031)$, which implies that the activity of the TBS in the forward orientation is greater than in the reverse orientation. Similarly, both the $5^{\prime} 1,292$ bp of the TBS (p1-TBSdel4) and the $3^{\prime} 1,191 \mathrm{bp}$ of the TBS (p1-TBSdel5) were able to impede enhancer-promoter interactions as evidenced by significantly decreased ( $P=0.010$ and $P=0.011$, respectively) levels of GUS activity in their leaves $(873.9 \pm 420.4$ and $1,536.4 \pm 758.3 \mathrm{pmol} \mathrm{MU} / \mathrm{min} \mathrm{mg}$ protein, respectively) compared to $\mathrm{p} 1$. However, the levels of GUS protein in the leaves of these lines were significantly higher $(P=0.013$ and $P=0.006$, respectively) than those lines containing the full-length $T B S$ in the forward orientation (p1-TBSF), which implies that the enhancer-blocking activities of the $5^{\prime} 1,292$ bp and $3^{\prime} 1,191$-bp fragments of the TBS were somewhat reduced compared with the full-length TBS sequence. 
(a)

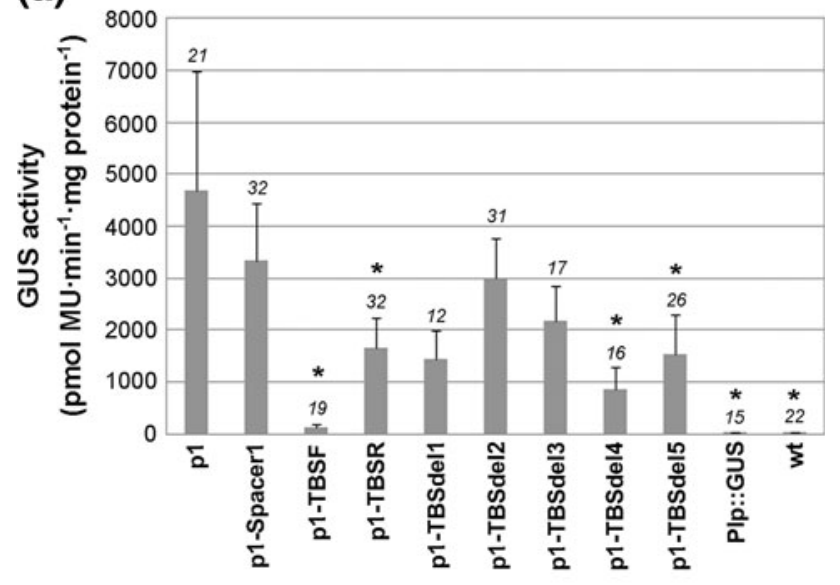

(b)

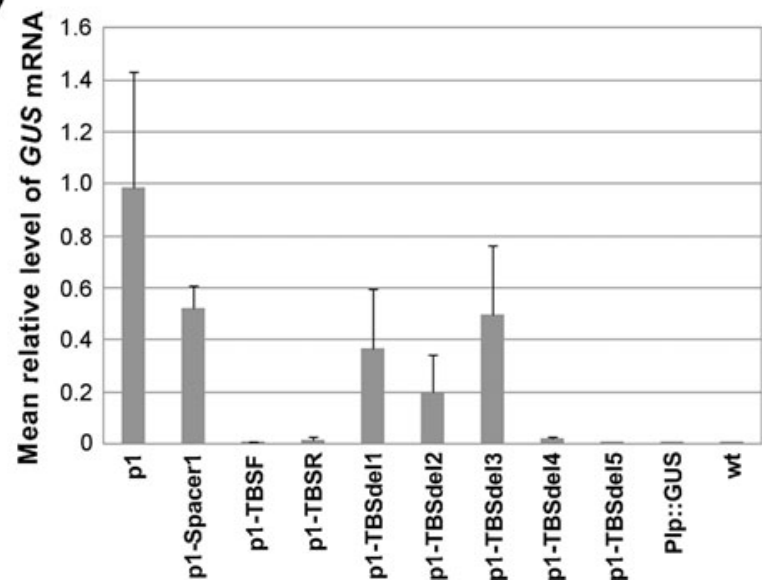

Fig. 3 Quantitative analyses of GUS activity in the leaves of transgenic Arabidopsis lines. a Each block represents the mean and standard error (bar) GUS activity measured fluorometrically in pmol MU generated minute ${ }^{-1} \mathrm{mg}_{\text {protein }}{ }^{-1}$ from three leaves of independent $T_{1}$ lines containing each construct respectively or untransformed controls. Italicized numbers above each block indicate the number of independent lines tested in each case. Asterisks denote mean values of GUS activity that are significantly reduced compared with that of lines containing the base vector $\mathrm{p} 1$, which lacks any insert. b Total RNA from the leaves of three independent replicate lines bearing each construct respectively, or the untransformed control, was assayed for levels of GUS transcript and the internal control, actin2. Each block represents the mean normalized value of GUS mRNA for each construct and bars indicate standard errors

Conversely, lines including the $5^{\prime} 827$ bp of the TBS (p1TBSdel1), the mid 465 bp of the TBS (p1-TBSdel2), and the $3^{\prime} 726 \mathrm{bp}$ of the TBS (p1-TBSdel3) displayed no significant difference $(P>0.05)$ from 1 lines in the level of GUS protein generated in leaf tissues $(1,432.3 \pm 5,50.9,3,011.8 \pm$ 765.3 and $2,169.8 \pm 672.5 \mathrm{pmol} \mathrm{MU} / \mathrm{min} \mathrm{mg}$ protein, respectively), suggesting that they were unable to considerably reduce the levels of enhancer-mediated activation of PIp-driven GUS expression in non-target tissues (Fig. 3a). However, in the case of lines bearing the p1-TBSdel1 construct, it is possible that this lack of significance may have been a direct result of the relatively small sample size of p1-TBSdel1 lines utilized in the fluorometric assays $(n=12)$.

Results of quantitative real-time RT-PCR analyses of GUS expression levels in the leaves of three randomly chosen lines containing each vector, respectively, as well as an untransformed control, were in agreement with histochemical and fluorometric GUS activity data (Fig. 3b). Lines bearing the p1-TBSF, p1-TBSR, p1-TBSdel4 and p1-TBSdel5 constructs exhibited very little GUS expression in the leaves compared with the remaining lines. These results further indicate that the TBS element is capable of inhibiting enhancer-promoter interference in both the forward and reverse orientations, and that both $5^{\prime}$ and $3^{\prime}$ fragments are sufficient to confer an enhancerblocking insulator function.

The TBS element functions as an enhancer-blocking insulator in multiple plant species and is not promoter-specific

To address whether the $T B S$ element is able to function as an enhancer-blocking insulator in another plant species, we transformed tobacco with a series of vectors containing the $35 S:: e G F P$ cassette in a head-to-head orientation with either the petal- and stamen-specific PIp-GUS cassette (Fig. 1a) or the phloem-specific AtSUS1p::GUS cassette (Fig. 1b). This allowed for the visual differentiation of enhancer-mediated expression (constitutive) from PIp- and AtSUS1p-mediated expression in vegetative tissues. In the case of the PIp-based vectors, histochemical analyses of GUS activity in petiole cross-sections indicated high levels of GUS staining in $56 \%$ of lines containing the p1 control vector $(n=9)$ and $100 \%$ of lines containing a $4-\mathrm{kb}$ 'inert' spacer sequence derived from bacteriophage $\lambda$ between the 35S::eGFP and PIp::GUS cassettes (p1-Spacer2; $n=7$; Fig. 4a). Conversely, none of the lines bearing the fulllength $T B S$ in the forward orientation (p1-TBSF; $n=4)$ exhibited any GUS staining in petiole tissues, which resembled PIp::GUS lines lacking the $35 S:: e G F P$ cassette $(n=8$; Fig. 4a). Similarly, $100 \%$ of positive control lines bearing the 35S::eGFP and AtSUS1p::GUS cassettes in a head-to-head orientation (p2;n=8) exhibited strong, constitutive GUS staining throughout the petiole. This was also the case in $89 \%$ of p2-Spacer2 lines, which contained the $4-\mathrm{kb} \lambda$ spacer sequence $(n=9)$. Conversely, $75 \%$ of lines lacking the 35S::eGFP cassette (AtSUS1p::GUS) displayed only phloem-specific expression in the petiole $(n=12)$, while the remainder $(25 \%)$ generated no GUS activity in the tissues analyzed. Insertion of the full-length TBS fragment in the forward orientation (p2-TBSF) resulted in phloem-specific expression in 54\% of the lines analyzed $(n=13)$, while the remaining lines exhibited some degree of leakiness in non-target tissues (Fig. 4b). 


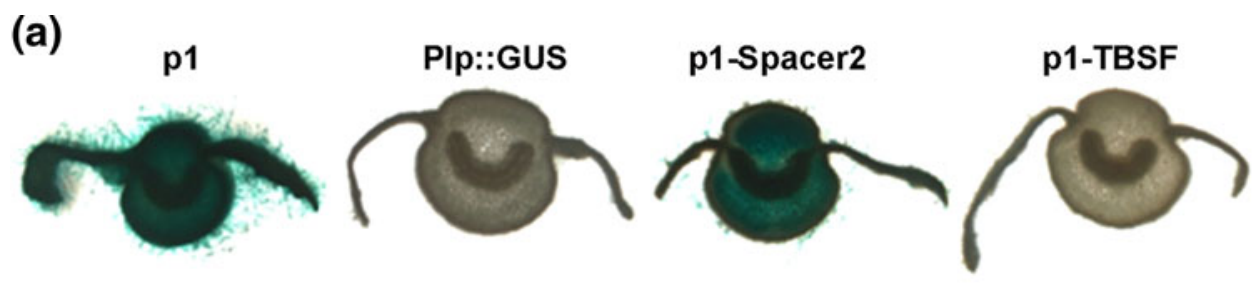

(b)
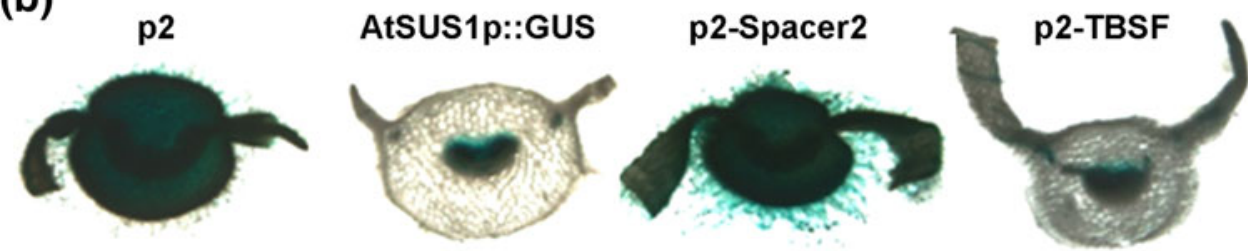

Fig. 4 Analysis of the enhancer-blocking insulator function of the $T B S$ in transgenic tobacco lines. a Representative petiole crosssections of lines transformed with various PIp::GUS derived constructs utilized to test the capability of the TBS element to impede constitutive $35 \mathrm{~S}$ enhancer-mediated activation of floral-

The TBS initiates transcription of a downstream GUS gene in both the forward and reverse orientations

It has been suggested previously that one possible mechanism by which enhancer-blocking insulators exert their function is through their inclusion of promoter-like sequences, which either act as a decoy by interacting directly with the enhancer, or through the attenuation of a signal that travels from enhancer to promoter (reviewed by Raab and Kamakaka 2010). To determine whether the TBS element possessed any promoter activity, we generated vectors in which full-length forward- and reverse-oriented TBS fragments were fused directly to a downstream GUS reporter gene (Fig. 1c), and transformed them into Arabidopsis. Histochemical assays of leaf and floral tissue from seven lines containing each vector, respectively, did not reveal any visible GUS staining, indicating that either the lines analyzed generated no functional GUS protein or the levels of GUS produced were too low to be detected using this method (Fig. 5a). In contrast, semiquantitative RT-PCR assays of GUS transcripts from a mixture of leaf and floral tissue from two independent lines bearing each vector, respectively, yielded weak amplification products in every case. Interestingly, the level of GUS expression appeared to be slightly higher when the $T B S$ was present in the reverse orientation (Fig. 5b).

In silico sequence analyses

Bioinformatic analysis of the TBS element for putative promoter regions (with a minimum cutoff score of 0.8 ) indicated the presence of a single putative promoter in each orientation. In the forward orientation, this included the region between nucleotides 533 and 583 with a

specific PIp in non-target tissues. b Petiole cross-sections of representative lines bearing AtSUS1p::GUS derived constructs designed to evaluate the ability of the $T B S$ element to block constitutive $35 S$ enhancer-induced activation of the phloem-specific AtSUS1 promoter

(a)

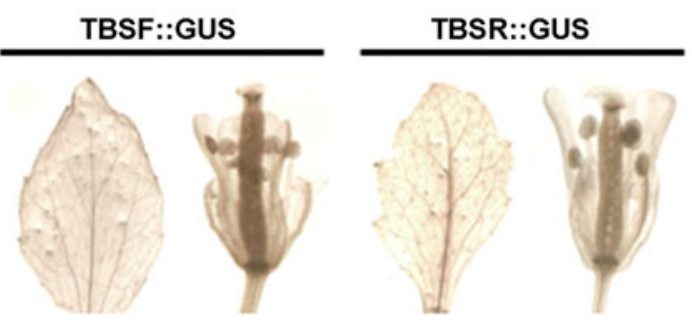

(b)

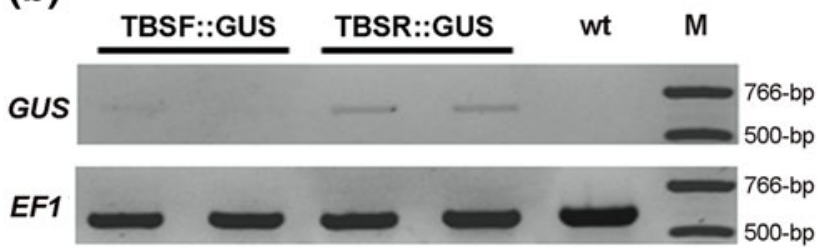

Fig. 5 Analysis of the TBS element for promoter-like activity. a Histochemical assays for GUS staining of leaves and flowers from representative Arabidopsis lines-bearing TBS-F::GUS (TBSF::GUS) and TBS-R::GUS (TBSR::GUS) cassettes, respectively. b Semiquantitative RT-PCR analysis of two representative TBSF::GUS and TBSR::GUS lines, respectively, along with an untransformed control, for $G U S$ expression in a mixture of leaf and floral tissues. EFl $\alpha$ was utilized as an internal control. The DNA marker is designated by ' $M$ '

transcription start site at nucleotide 574 (score of 0.91). In the reverse orientation, the putative promoter region occurred between nucleotides 791 and 741 with a transcription start site at nucleotide 750 (score of 0.80).

\section{Discussion}

The need for techniques with which to reduce enhancerpromoter interference in transgenic plants has become a 
necessity in recent years due to the increased use of a more comprehensive approach to genetic engineering in which transgenic constructs containing several transcriptional units are utilized for the simultaneous improvement of multiple agronomic traits. Several methods have been proposed to prevent such interactions, including the inclusion of a spacer DNA fragment between enhancer and promoter, or the use of promoters that contain only weak enhancers. However, these techniques have been shown to be relatively unpredictable and their effectiveness often varies from construct to construct (Gudynaite-Savitch et al. 2009). Another means of averting enhancer-promoter communication in transgenic constructs is the use of enhancer-blocking insulators, which impede such interactions when situated between enhancer and promoter, and are commonly used during mammalian cell transfection experiments (Steinwaerder and Lieber 2000; Ye et al. 2003). Unfortunately, relatively little is known concerning these elements in plants; however, several sequences exhibiting enhancer-blocking activity in plants have been identified in recent years (Gudynaite-Savitch et al. 2009; Hily et al. 2009; Singer et al. 2010b; van der Geest and Hall 1997). One such sequence is the TBS fragment from $P$. hybrida, which has been shown to impede inappropriate enhancer-promoter interference in transgenic Arabidopsis when situated in the reverse orientation (relative to the sequence deposited in GenBank) between the $35 \mathrm{~S}$ enhancer and the flower-specific AGIP target promoter (Hily et al. 2009). In this study, we further characterized the enhancerblocking function of this element in an attempt to gain insight into the mechanism behind its activity.

To determine whether the activity of the TBS element was dependent on its polarity, we inserted the full-length fragment in both the forward and reverse orientations, respectively, between the partially duplicated $35 S$ enhancer and the petal- and stamen-specific PIp::GUS cassette (Fig. 1a), and transformed the resulting vectors into Arabidopsis. We found that the TBS element was capable of reducing enhancer-promoter interference in both orientations (Fig. 2a); however, quantitative analyses of GUS activity in leaf tissues (where the PIp is not active unless it is activated by the constitutive $35 \mathrm{~S}$ enhancer) indicated that the strength of the insulator appeared to be significantly $(P<0.05)$ higher when present in the forward orientation (Fig. 3a). Conversely, only a small difference was noted between the levels of GUS transcript in the leaves of p1-TBSF and p1-TBSR lines (Fig. 3b), but this may have been due to the smaller sample size (three independent lines containing each vector) utilized for quantitative RT-PCR analyses. Interestingly, a recent comparison of the insulation capabilities of the TBS element and a $1-\mathrm{kb}$ fragment of bacteriophage lambda, which has also been found to confer enhancer-blocking function in Arabidopsis
(Singer et al. 2010b), revealed that the TBS was the least effective of the two (Yang et al. 2011). However, as was the case in the original study of the insulation function of the $T B S$ element (Hily et al. 2009), the $T B S$ was inserted between enhancer and target promoter in the reverse, and seemingly less efficient, orientation. Therefore, it is likely that the TBS fragment is capable of providing a more effective means of blocking enhancer-blocking communication than previously thought.

While many enhancer-blocking insulators do not appear to exhibit orientation-dependency (for example Tchurikov et al. 2009), this is not the first case in which such an element has been found to exhibit functional polarity (Abhyankar et al. 2007; Barges et al. 2000; Bell and Felsenfeld 2000; Hark et al. 2000). Unfortunately, the mechanism behind such an effect remains elusive. One possible reason for this type of polar behavior is the fact that insulators often also contain additional regulatory elements due to their length. One such regulatory element that has been found in very close proximity to an insulating sequence is an enhancer element, as is the case for the enhancer-blocking insulator situated upstream of the human apoB locus (Antes et al. 2001). Since enhancerblocking insulators are known to be functional only when situated between an enhancer and promoter, compound elements comprising both enhancer-blocking and enhancer activities would almost certainly display polarity. In such a case, orientation of the insulator-enhancer element with the insulator proximal to the target promoter would block both the internal and external enhancers, while a reversed orientation would still block any external enhancers, but not that contained within the insulator (reviewed by West et al. 2002).

Indeed, it would not be wholly unexpected for the fulllength $T B S$ fragment to contain other regulatory elements given its relatively large size ( $2 \mathrm{~kb})$. Intriguingly, we found the TBS element to be capable of driving expression of a downstream GUS reporter gene (Fig. 5b). GUS expression was never noted in the same RT-PCR assay using total RNA from lines containing a promoterless GUS reporter gene (data not shown), which suggests that the observed transcripts were not simply due to positional effects of the construct in the genome. While GUS-specific transcripts were present in these transgenic lines, no functional GUS protein was detected (Fig. 5a), which is not surprising, since transcription initiation could have occurred at virtually any site within the 2-kb TBS fragment leading to the generation of non-functional transcripts. Several recent studies have shown that a wide variety of enhancers can initiate transcription autonomously in a range of organisms (Dobi and Winston 2007; Kim et al. 2007; Ling et al. 2005; Routledge and Proudfoot 2002; Tuan et al. 1992; Singer et al. 2010a), which raises the possibility that the observed 
$G U S$-specific transcripts initiated by the $T B S$ element in this study result from the presence of a cryptic enhancer element within the full-length $T B S$ fragment. Furthermore, since enhancers function in an orientation-independent manner (for example Banerji et al. 1981), this enhancerinitiated transcription is likely bi-directional, which would correspond to the fact that transcription of a downstream reporter gene was observed when the TBS was in either orientation, but at a seemingly higher level in the reverse orientation (Fig. 5b). Therefore, one could speculate that the $T B S$, like the human $a p o B$ insulator, is a composite element containing an internal enhancer upstream of the insulator, which would potentially explain its polarity.

Alternatively, in light of the fact that the TBS appears to initiate transcription of a downstream GUS reporter gene in transgenic Arabidopsis in both orientations (Fig. 5), and that putative promoter regions were identified using bioinformatic tools in both orientations, it is possible that this sequence contains promoter activity that contributes toward its function as an enhancer-blocking insulator. While it appears that several different mechanisms may exist by which enhancer-blocking insulators function, it has been suggested that the addition of a promoter upstream of the target promoter can result in trapping of the enhancer and will prevent transcriptional activation of the target promoter (Geyer 1997). Similarities have been found to exist between insulators and promoters, including distinct chromatin modification signatures, the binding of specific transcription factors and localization to particular nuclear regions (reviewed by Raab and Kamakaka 2010), and several Drosophila insulators have been found to contain promoters (Bae et al. 2002; Drewell et al. 2002; Geyer 1997). Recently, it has been shown that promoters containing stalled polymerase II are more likely to display enhancer-blocking insulator activity than non-stalled promoters in Drosophila (Cande et al. 2009; Chopra et al. 2009), which may result from enhancer preference for components of the stalled transcriptional complex or an inherent selectivity of enhancers for particular types of promoters (Butler and Kadonaga 2001; Juven-Gershon et al. 2008). It is possible that a similar scenario is occurring in this system; however, further studies will be required to ascertain whether this is the case with the $T B S$ element.

Intriguingly, while both the overlapping $5^{\prime}$ 1,292 bp (p1-TBSdel4) and $3^{\prime}$ 1,191 bp (p1-TBSdel5), and to a lesser extent possibly the $5^{\prime} 827 \mathrm{bp}$ (p1-TBSdel1), of the $T B S$ were able to reduce enhancer-promoter communication in transgenic Arabidopsis (Fig. 2b), neither fragment achieved the effectiveness of the full-length insulator (Fig. 3), which suggests that there may be an additive effect of several elements within the full-length $T B S$. This is similar to the well-characterized Drosophila insulator, gypsy, which comprises a cluster of 12 repeats that are bound by the zinc finger Suppressor of Hairy-wing $[\mathrm{Su}(\mathrm{Hw})]$ protein (Spana et al. 1988). At least four tightly spaced $\mathrm{Su}(\mathrm{Hw})$ binding sites are necessary for effective enhancer-blocking function (Scott et al. 1999) and truncated versions have been shown to only possess weak enhancer-blocking activity (Hagstrom et al. 1996). Therefore, it stands to reason that the TBS contains multiple elements that are required in combination to exert its full activity.

The full-length, forward-oriented, TBS fragment has also been found to be effective for impeding enhancerpromoter interference in transgenic tobacco plants, and is not promoter-specific, suggesting that it may be applicable for use in a broad range of plant species (Fig. 4). This resembles the case of many metazoan insulators, which have been found to function in a variety of organisms (Chung et al. 1993; Namciu et al. 1998). Similarly, it has recently been found that the BEAD-1 and BEAD-1C insulators from the human T-cell receptor $\alpha / \delta$ locus (Zhong and Krangel 1997), as well as the $\mathrm{UAS}_{\text {rpg }}$ insulator from Ashbya gossypii (Bi and Broach 2006), reduce non-specific enhancer-promoter interactions in transgenic Arabidopsis (Gudynaite-Savitch et al. 2009), which hints at the possibility of conserved mechanisms between an array of eukaryotic organisms.

In conclusion, there is an imminent requirement for effective tools with which to block inappropriate interactions between enhancers and promoters in plant transformation vectors due to ever-increasing reports of mis-expression of transgenes as a result of enhancer-promoter interference. While further research is still needed to elucidate the exact mechanism behind both enhancerpromoter interactions and enhancer-blocking insulators in plants, the identification of promoter/enhancer activity in the recently identified $T B S$ insulator provides additional insight into this matter. Also, the ability of the forwardoriented TBS insulator to protect a range of tissue-specific plant promoters from the strong, constitutive $35 \mathrm{~S}$ enhancer in multiple plant species provides further evidence for its potential use as a practical tool in the generation of transgenic crops in the future.

Acknowledgments The authors wish to thank Sara Villani for her invaluable technical assistance and Dr. Zongrang Liu (ARS-USDA, Kearneysville, WV) for his generosity in supplying a sample of the TBS element. This study was supported by state, federal, and institutional funds appropriated to the New York State Agricultural Experiment Station, Cornell University.

\section{References}

Abhyankar MM, Urekar C, Reddi PP (2007) A novel CpG-free vertebrate insulator silences the testis-specific $S P-10$ gene in somatic tissues. J Biol Chem 282:36143-36154 
Alonso JM, Stepanova AN, Leisse TJ, Kim CJ et al (2003) Genomewide insertional mutagenesis of Arabidopsis thaliana. Science 301:653-657

Antes TJ, Namciu SJ, Fournier REK, Levy-Wilson B (2001) The $5^{\prime}$ boundary of the human apolipoprotein B chromatin domain in intestinal cells. Biochemistry 40:6731-6742

Bae E, Calhoun VC, Levine M, Lewis EB, Drewell RA (2002) Characterization of the intergenic RNA profile at abdominal$A$ and Abdominal-B in the Drosophila bithorax complex. Proc Natl Acad Sci 99:16847-16852

Banerji J, Rusconi S, Schaffner W (1981) Expression of a beta-globin gene is enhanced by remote SV40 DNA sequences. Cell 2:299-308

Barges S, Mihaly J, Galloni M, Hagstrom K, Müller M, Shanower G, Schedl P, Gyurkovics H, Karch F (2000) The Fab-8 boundary defines the distal limit of the bithorax complex $i a b-7$ domain and insulates $i a b-7$ from initiation elements and a PRE in the adjacent $i a b-8$ domain. Development 127:779-790

Bell AC, Felsenfeld G (2000) Methylation of a CTCF-dependent boundary controls imprinted expression of the Igf2 gene. Nature 405:482-485

Bi X, Broach JR (2006) UAS $_{\text {rpg }}$ can function as a heterochromatin boundary element in yeast. Genes Dev 13:1089-1101

Butler JE, Kadonaga JT (2001) Enhancer-promoter specificity mediated by DPE or TATA core promoter motifs. Gene Dev 15:2515-2519

Cande JD, Chopra VS, Levine M (2009) Evolving enhancer-promoter interactions within the tinman complex of the flour beetle, Tribolium castaneum. Development 136:3153-3160

Charrier B, Champion A, Kreis M (2002) Expression profiling of the whole Arabidopsis Shaggy-like kinase multigene family by realtime reverse transcriptase-polymerase chain reaction. Plant Physiol 130:1-14

Chopra VS, Cande J, Hong JW, Levine M (2009) Stalled Hox promoters as chromosomal boundaries. Genes Dev 23:15051509

Chung JH, Whiteley M, Felsenfeld G (1993) A $5^{\prime}$ element of the chicken $\beta$-globin domain serves as an insulator in human erythroid cells and protects against position effect in Drosophila. Cell 74:505-514

Clough SJ, Bent AF (1998) Floral dip: a simplified method for Agrobacterium-mediated transformation of Arabidopsis thaliana. Plant J 16:735-743

Dobi KC, Winston F (2007) Analysis of transcriptional activation at a distance in Saccharomyces cerevisiae. Mol Cell Biol 27:55755586

Drewell RA, Bae E, Burr J, Lewis EB (2002) Transcription defines the embryonic domains of cis-regulatory activity at the Drosophila bithorax complex. Proc Natl Acad Sci USA 99:1685316858

Gaszner M, Felsenfeld G (2006) Insulators: exploiting transcriptional and epigenetic mechanisms. Nat Rev Genet 7:703-713

Geyer PK (1997) The role of insulator elements in defining domains of gene expression. Curr Opin Genet Dev 7:242-248

Geyer PK, Spana C, Corces VG (1986) On the molecular mechanism of gypsy-induced mutations at the yellow locus of Drosophila melanogaster. EMBO J 5:2657-2662

Goderis IJWM, De Bolle MFC, François IEJA, Wouters PFJ, Broekaert WF, Cammue BPA (2002) A set of modular plant transformation vectors allowing flexible insertion of up to six expression units. Plant Mol Biol 50:17-27

Gudynaite-Savitch L, Johnson DA, Miki BLA (2009) Strategies to mitigate transgene-promoter interactions. Plant Biotechnol J 7:472-485

Hagstrom K, Muller M, Schedl P (1996) Fab-7 functions as a chromatin domain boundary to ensure proper segment specification by the Drosophila bithorax complex. Genes Dev 10:3202-3215

Hajdukiewicz P, Svab Z, Maliga P (1994) The small, versatile $p P Z P$ family of Agrobacterium binary vectors for plant transformation. Plant Mol Biol 25:989-994

Hark AT, Schoenherr CJ, Katz DJ, Ingram RS, Levorse JM, Tilghman SM (2000) CTCT mediates methylation-sensitive enhancerblocking activity at the H19/Igf2 locus. Nature 405:486-489

Hily JM, Liu Z (2009) A simple and sensitive high-throughput GFP screening in woody and herbaceous plants. Plant Cell Rep 28:493-501

Hily JM, Singer SD, Yang Y, Liu Z (2009) A transformation booster sequence $(T B S)$ from Petunia hybrida functions as an enhancerblocking insulator in Arabidopsis thaliana. Plant Cell Rep 28:1095-1104

Honma T, Goto K (2000) The Arabidopsis floral homeotic gene PISTILLATA is regulated by discrete cis-elements responsive to induction and maintenance signals. Development 127:2021-2030

Horsch RB, Fry JE, Hoffmann NL, Wallroth M, Eichholtz D, Rogers SG, Fraley RT (1985) A simple and general method for transferring genes into plants. Science 227:1229-1231

Jagannath A, Bandyopadhyay P, Arumugam N, Gupta V, Kumar P, Pental D (2001) The use of a Spacer DNA fragment insulates the tissue-specific expression of a cytotoxic gene (barnase) and allow high-frequency generation of transgenic male sterile lines in Brassica juncea $\mathrm{L}$. Mol Breeding 8:11-23

Jefferson RA, Kavanagh TA, Bevan MW (1987) GUS fusions: $\beta$-glucuronidase as a sensitive and versatile gene fusion marker in higher plants. EMBO J 6:3901-3907

Juven-Gershon T, Hsu JY, Kadonaga JT (2008) Caudal, a key developmental regulator, is a DPE-specific transcriptional factor. Genes Dev 22:2823-2830

Kadauke S, Blobel GA (2009) Chromatin loops in gene regulation. Biochim Biophys Acta 1789:17-25

Kay R, Chan A, Daly M, McPherson J (1987) Duplication of CaMV $35 \mathrm{~S}$ promoter sequences creates a strong enhancer for plant genes. Science 236:1299-1302

Kellum R, Schedl P (1992) A group of scs elements function as domain boundaries in an enhancer-blocking assay. Mol Cell Biol 12:2424-2431

Kim A, Zhao H, Ifrim I, Dean A (2007) $\beta$-globin intergenic transcription and histone acetylation dependent on an enhancer. Mol Cell Biol 27:2980-2986

Ling J, Baibakov B, Pi W, Emerson BM, Tuan D (2005) The HS2 enhancer of the $\beta$-globin locus control region initiates synthesis of non-coding, polyadenylated RNAs independent of a cis-linked globin promoter. J Mol Biol 350:883-896

Murashige T, Skoog F (1962) A revised medium for rapid growth and bioassays with tobacco tissue cultures. Physiol Plantarum 15:473-497

Namciu SJ, Blochlinger KB, Fournier REK (1998) Human matrix attachment regions insulate transgene expression from chromosomal position effects in Drosophila melanogaster. Mol Cell Biol 18:2382-2391

Odell JT, Knowlton S, Lin W, Mauvais J (1988) Properties of an isolated transcription stimulating sequence derived from the cauliflower mosaic virus 35S promoter. Plant Mol Biol 10:263-272

Ohta S, Mita S, Hattori T, Nakamura K (1990) Construction and expression in tobacco of a $\beta$-glucuronidase (GUS) reporter gene containing an intron within the coding sequence. Plant Cell Physiol 31:805-813

Raab JR, Kamakaka RT (2010) Insulators and promoters: closer than we think. Nat Rev Genet 11:439-446

Reese MG (2001) Application of a time-delay neural network to promoter annotation in the Drosophila melanogaster genome. Comput Chem 26:51-56 
Rosso MG, Li Y, Strizhov N, Reiss B, Dekker K, Weisshaar B (2003) An Arabidopsis thaliana T-DNA mutagenized population (GABI-Kat) for flanking sequence tag-based reverse genetics. Plant Mol Biol 53:247-259

Routledge SJE, Proudfoot NJ (2002) Definition of transcriptional promoters in the human $\beta$ globin locus control region. J Mol Biol 323:601-611

Sadeghi A, Broeders S, De Greve H, Hernalsteens J-P, Peumans WJ, Van Damme EJM, Smagghe G (2007) Expression of garlic leaf lectin under the control of the phloem-specific promoter Asus 1 from Arabidopsis thaliana protects tobacco plants against the tobacco aphid (Myzus nicotianae). Pest Manag Sci 63:1215-1223

Scott KC, Taubman AD, Geyer PK (1999) Enhancer blocking by the Drosophila gypsy insulator depends upon insulator anatomy and enhancer strength. Genetics 153:787-798

Singer SD, Cox KD, Liu Z (2010a) Both the constitutive Cauliflower Mosaic Virus $35 S$ and tissue-specific AGAMOUS enhancers activate transcription autonomously in Arabidopsis thaliana. Plant Mol Biol 74:293-305

Singer SD, Hily J-M, Liu Z (2010b) A 1 kb bacteriophage lambda fragment functions as an insulator to effectively block enhancerpromoter interactions in Arabidopsis thaliana. Plant Mol Biol Rep 28:69-76

Singer SD, Cox KD, Liu Z (2011) Enhancer-promoter interference and its prevention in transgenic plants. Plant Cell Rep 30:723-731

Spana C, Harrison DA, Corces VA (1988) The Drosophila melanogaster suppressor of Hairy-wing protein binds to specific sequences of the gypsy retrotransposon. Genes Dev 2:1414-1423

Steinwaerder DS, Lieber A (2000) Insulation from viral transcriptional regulatory elements improves inducible transgene expression from adenovirus vectors in vitro and in vivo. Gene Ther 7:556-567
Tchurikov NA, Kretova OV, Moiseeva ED, Sosin DV (2009) Evidence for RNA synthesis in the intergenic region between enhancer and promoter and its inhibition by insulators in Drosophila melanogaster. Nucleic Acids Res 37:111-122

Tuan D, Kong S, Hu K (1992) Transcription of the hypersensitive site HS2 enhancer in erythroid cells. Proc Natl Acad Sci USA 89:11219-11223

van der Geest AHM, Hall TC (1997) The $\beta$-phaseolin $5^{\prime}$ matrix attachment region acts as an enhancer facilitator. Plant Mol Biol 33:553-557

van Engelen FA, Molthoff JW, Conner AJ, Nap J-P, Pereira A, Stiekema WJ (1995) pBINPLUS: an improved plant transformation vector based on pBIN19. Transgenic Res 4:288-290

Wallace JA, Felsenfeld G (2007) We gather together: insulators and genome organization. Curr Opin Genet Dev 17:400-407

West AG, Gaszner M, Felsenfeld G (2002) Insulators: many functions, many mechanisms. Genes Dev 16:271-288

Yang Y, Singer SD, Liu Z (2011) Evaluation and comparison of the insulation efficiency of three enhancer-blocking insulators in plants. Plant Cell Tiss Org 105:405-414

Ye X, Liang M, Meng X, Ren X, Chen H, Li ZY, Ni S, Lieber A, $\mathrm{Hu} F$ (2003) Insulation from viral transcriptional regulatory elements enables improvement to hepatoma-specific gene expression from adenovirus vectors. Biochem Biophys Res Commun 307:759-764

Zheng X, Deng W, Luo K, Duan H, Chen Y, McAvoy R, Song S, Pei Y, Li Y (2007) The cauliflower mosaic virus (CaMV) $35 \mathrm{~S}$ promoter sequence alters the level and patterns of activity of adjacent tissue- and organ-specific gene promoters. Plant Cell Rep 26:1195-1203

Zhong X-P, Krangel MS (1997) An enhancer-blocking element between $\alpha$ and $\delta$ gene segments within the human T cell receptor $\alpha / \delta$ locus. Proc Natl Acad Sci 94:5219-5224 